\title{
Wavelet-Packet Identification of Dynamic Systems with Coloured Measurement Noise
}

\author{
Henrique Mohallem Paiva ${ }^{1}$ and Roberto Kawakami Harrop Galvão ${ }^{2}$ \\ ${ }^{1}$ Empresa Brasileira de Aeronáutica - EMBRAER \\ São José dos Campos, SP, 12227-901, Brazil \\ henrique.paiva@embraer.com.br \\ 2 Instituto Tecnológico de Aeronáutica - ITA \\ CTA, São José dos Campos, SP, 12228-900, Brazil \\ kawakami@ele.ita.br
}

\begin{abstract}
This paper analyses the effect of coloured noise on a recently proposed technique for linear system identification in frequency subbands using wavelet packets. For this purpose, a simulation study involving the longitudinal dynamics of a flexible aircraft model is presented. The results reveal that the wavelet-packet identification outcome is robust with respect to changes in the spectral noise features. In particular, the identified frequency response is effectively smoothed in regions with poor signal-to-noise ratio. Finally, the results are favourably compared, in terms of resonance peak identification, with those obtained by standard time-domain identification methods.
\end{abstract}

\section{Introduction}

Identification techniques may be a useful alternative to physical modelling of dynamic systems when the underlying phenomena are complex or when the direct measurement of model parameters is not practical. In many cases, linear identification can generate adequate models for engineering purposes, provided that the system is operated within a suitable working range 12 .

In recent years, there has been growing interest in applications of the Wavelet Transform for linear system identification. Wavelets have been employed to characterize spectrally rich impulse responses with long subsequences of small coefficients [9], which arise in problems such as echo canceling in communication networks. Moreover, wavelets have been used to represent both slow and fast parameter variations in the identification of time-varying systems, such as communication channels to be equalized [17. A wavelet model particularly suitable for adaptive identification of linear periodic time-varying systems was presented in [6. Several applications can also be found in the context of mechanical systems, such as modal parameter identification [7]1] and vibration signal modelling for fault diagnosis purposes 3 .

It is worth noting that most research efforts concerning the use of wavelet decompositions in linear system identification have focused on multiscale octaveband and single-level M-band decomposition 15. Wavelet-packet algorithms, 
which are popular in other signal processing areas such as audio/image coding 14] and pattern recognition [10], have not been common in this context. This gap was addressed by a recent paper [13, in which wavelet packet decomposition trees were employed to establish frequency bands for the identification of subband models. The tree structure was optimized by using a generalized cross-validation method in order to achieve a compromise between accuracy and parsimony of the overall model. In comparison with a standard ARX (autoregressive with exogenous input) identification method, the proposed technique was found to be superior in terms of resonance peak identification and sensitivity to white measurement noise. However, investigations concerning coloured noise were not carried out.

Appropriate treatment of coloured noise, which is the most common form of noise in practical situations 15, may be essential to obtain suitable models by system identification, as discussed elsewhere [81618]. The present work investigates the effect of coloured measurement noise on the performance of the wavelet-packet identification algorithm proposed in [13. For this purpose, a case study involving the dynamics of a flexible aircraft model is presented. Inputoutput data are generated by numerical simulations with the inclusion of additive measurement noise within a particular frequency region. The effect of the spectral noise features on the identification outcome is investigated by varying the frequency region in which the measurements are degraded. The wavelet-packet identification results are compared with those obtained by standard ARX and ARMAX (auto-regressive moving-average with exogenous input) methods.

The remaining sections of this paper are organized as follows. Section 2 briefly describes the wavelet-packet identification technique under consideration. The case study is presented in section 3. Finally, concluding remarks are given in section 4.

\section{The Wavelet-Packet Identification Technique}

This section presents a brief review of the wavelet-packet identification technique proposed in [13. For this purpose, some notation details will be initially introduced. Let $\mathrm{H}$ and $\mathrm{G}$ be the lowpass and highpass filters associated to the wavelet-packet decomposition tree, respectively, and $H_{r}$ and $G_{r}$ be the associated reconstruction filters [15]. In the present work, these filters are adopted such that the filter bank is orthonormal. $\downarrow 2$ and $\uparrow 2$ denote downsampling and upsampling operations, respectively. $\mathbf{y}$ is the signal to be decomposed. $\mathbf{x}_{i, j}\{\mathrm{y}\}$ represents the wavelet coefficients of signal $\mathbf{y}$ at node $(i, j)$ of the wavelet tree, where $j \geq 0$ indicates the resolution level and $i$ is a sequential index ranging from 0 to $2^{j}-1$. Node $(i, j)$ is the parent of nodes $(2 i, j+1)$ and $(2 i+1, j+1)$, which are the children of node $(i, j)$. All nodes without children are called leaf nodes. The depth of the tree is defined as the highest level in which nodes are present. A tree is said to be complete if all leaf nodes are in the same resolution level. Scalars and row vectors are represented by italic and boldface lowercase symbols, respectively. 


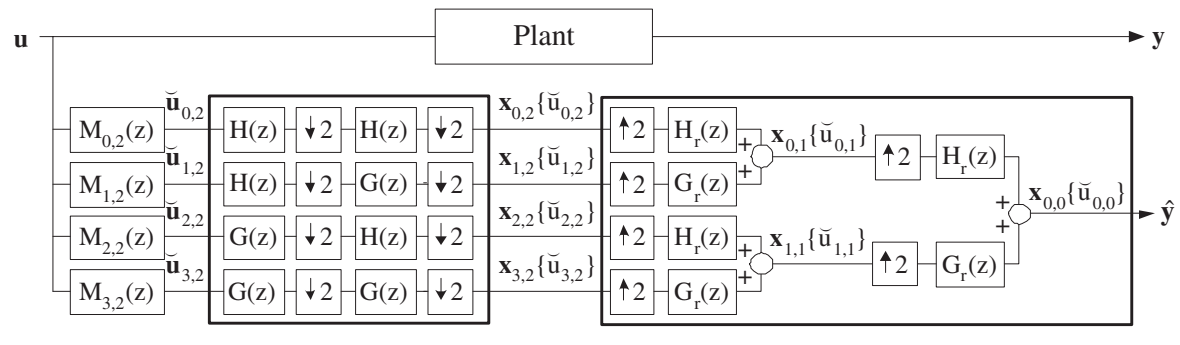

Wavelet-Packet Decomposition Tree (with multiple inputs)

Fig. 1. Example of the system modelling scheme for a particular wavelet-packet decomposition tree with four leaf nodes (in this example, all leaf nodes are in the same level, but this is not a requirement)

The identification algorithm is based on the development of several subband models. The wavelet-packet decomposition tree is used to establish the frequency bands at which the subband models will be created. Each leaf node of the tree is associated to a frequency band, and the complete set of leaf nodes composes the whole frequency range. For each frequency band, a subband model is created.

This modelling scheme is illustrated in Fig. 1 for a particular structure of the wavelet-packet decomposition tree. In this figure, $\mathbf{M}_{i, j}$ indicates the subband model intended to represent the plant in the frequency band associated to the leaf node $(i, j)$. If $(i, j)$ is a leaf node, then signal $\breve{\mathbf{u}}_{i, j}$ is defined as the output of model $\mathrm{M}_{i, j}$ for input $\mathbf{u}$. If $(i, j)$ is not a leaf node, then the coefficients $\mathbf{x}_{i, j}\left\{\breve{\mathbf{u}}_{i, j}\right\}$ are defined as the reconstruction of the coefficients at the children nodes of $(i, j)$.

The structure adopted for each subband model is a transfer function of the form of the form $\mathrm{M}(\mathrm{z})=\left(1-\mathrm{z}^{-1}\right)^{-s}\left(\alpha+\beta \mathrm{z}^{-1}\right), s \in \mathbb{Z}, \alpha, \beta \in \mathbb{R}$.

Parameters $s_{i, j}, \alpha_{i, j}$ and $\beta_{i, j}$ are estimated in order to minimize the cost function $J_{i, j}\left(s_{i, j}, \alpha_{i, j}, \beta_{i, j}\right)=\mathbf{e}_{i, j}\left(\mathbf{e}_{i, j}\right)^{\mathrm{T}}$, where residue $\mathbf{e}_{i, j}=\mathbf{x}_{i, j}\left\{\mathrm{y}-\breve{u}_{i, j}\right\}$ denotes the wavelet-packet coefficients of the difference between the plant output $\mathbf{y}$ and the subband model output $\breve{\mathbf{u}}_{i, j}$, in the frequency band under consideration.

For a fixed value of $s_{i, j}$, cost $J_{i, j}$ is minimized with respect to $\alpha_{i, j}$ and $\beta_{i, j}$ by a least-squares procedure. A search algorithm is used to find the value of $s_{i, j}$ that leads to the minimum value of $J_{i, j}$. The details of these procedures are described in [13].

As demonstrated in [13], the cost $J_{0,0}$, associated to the root node $(i, j)=$ $(0,0)$ of the wavelet-packet tree, is equal to the square of the 2-norm of the prediction error $(\mathbf{y}-\hat{\mathbf{y}})$, that is, $J_{0,0}=(\mathbf{y}-\hat{\mathbf{y}})(\mathbf{y}-\hat{\mathbf{y}})^{\mathrm{T}}$. Furthermore, for orthonormal wavelets, the cost at a non-leaf node $(i, j)$ was shown to be equal to the sum of the costs at its children nodes, i.e., $J_{i, j}=J_{2 i, j+1}+J_{2 i+1, j+1}$. Such features allow the use of the following algorithm to choose the best wavelet-packet tree, which presents the minimum 2-norm of the prediction error [13]. 
1 - Fix the maximum depth $d$ allowed for the tree and initialize the search with a complete tree with that depth.

2 - All nodes are candidates to be leaf nodes. Thus, for each node $(i, j)$, leaf or non-leaf, obtain the associated subband model $\mathrm{M}_{i, j}(\mathrm{z})$. Calculate, for each node, the square of the 2 -norm of the residue $\mathbf{e}_{i, j}$ and call this value $J_{i, j}^{l}$. Superscript $l$, standing for leaf, is used to emphasize that $J_{i, j}^{l}$ will be equal to the cost $J_{i, j}$ at node $(i, j)$ only if this node is chosen to be a leaf.

3 - Initialize the costs at level $d$, the deepest level of the tree. If one node of this level is kept in the tree, it will be necessarily a leaf node. Thus, let $J_{i, d}=J_{i, d}^{l}$, for all $i=0,1, \ldots,\left(2^{d}-1\right)$.

4 - Analyze the other nodes of the tree, which can be made either leaf or non-leaf nodes. Start from level $j=d-1$ and use a bottom-up approach (that is, analyze all nodes of a level before evaluating the parent level). Decide if each node $(i, j)$ should be a leaf or a non-leaf node, by comparing cost $J_{i, j}^{l}$ (cost if $(i, j)$ is a leaf node) with the sum $J_{2 i, j+1}+J_{2 i+1, j+1}$ of the costs at its children nodes (cost if $(i, j)$ is a non-leaf node). The decision rule is:

$$
J_{i, j}=\left\{\begin{array}{l}
J_{i, j}^{l}, \text { if } J_{i, j}^{l} \leq \rho\left(J_{2 i, j+1}+J_{2 i+1, j+1}\right) \\
J_{2 i, j+1}+J_{2 i+1, j+1}, \text { otherwise }
\end{array}\right.
$$

where the penalty factor $\rho \geq 1$ ensures that node $(i, j)$ will only be split into children nodes if the cost reduction is large enough to justify the increase in model complexity.

The penalty factor $\rho$ is required to avoid an overfitting of the identification data. An increase in the value of $\rho$ tends to reduce the number of nodes in the resulting tree. Thus, the choice of the penalty factor $\rho$ can be regarded as a model order determination problem, which is addressed by using a generalized cross validation (GCV) method, described in detail in [13. The value of $\rho$ that minimizes the GCV index (GCVi) is selected, thus providing a tradeoff between model parsimony and identification accuracy. Therefore, the ability of the model to represent the behaviour of the system for input signals different from the one used in the identification is improved.

\section{Case Study}

This case study is concerned with the identification of a longitudinal flexible model for a simulated aircraft in straight flight [2]. This example was employed in [13] to illustrate the performance of the wavelet-packet technique in the presence of white measurement noise. The continuous-time transfer function adopted in the simulation is presented in equation (2), where the input and output variables indicate the elevator angle and the aircraft pitch angle measured by the sensor, respectively, both in degrees. In the simulation, this transfer function was discretized at a sampling rate of $40 \mathrm{~Hz}$.

$$
\frac{Y(s)}{U(s)}=\frac{0.4062 \mathrm{~s}^{3}-2.034 \mathrm{~s}^{2}+444.2 \mathrm{~s}-2015}{\mathrm{~s}^{4}+1.81 \mathrm{~s}^{3}+1925 \mathrm{~s}^{2}+1822 \mathrm{~s}+890400}
$$




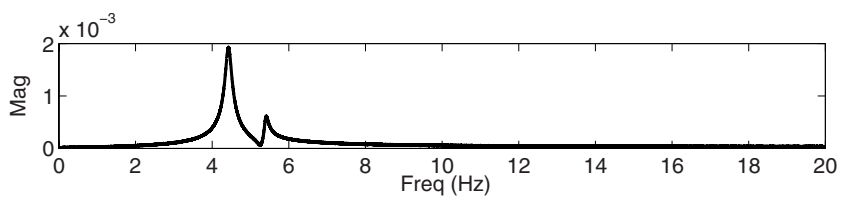

Fig. 2. Fast Fourier Transform of the plant output without measurement noise
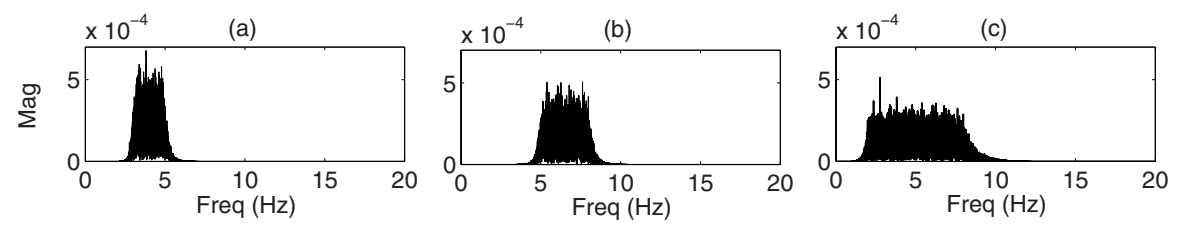

Fig. 3. Fast Fourier Transform of the measurement noise in the frequency bands (a) (3-5) $\mathrm{Hz},(\mathrm{b})(5-8) \mathrm{Hz}$, and (c) (2-8) $\mathrm{Hz}$

The following chirp excitation was used for identification: $u[k T]=\sin (2 \pi f k T)$, where $k=0,1, \ldots, 10^{4}, T=0.025 \mathrm{~s}$, and frequency $f$ varies linearly from 0.1 to 10 Hz. Fig. 2 presents the Fast Fourier Transform of the resulting output signal.

The $d b 8$ filters from the Daubechies family were adopted, as in [13. The maximum depth of the decomposition tree was set to six. For comparison, ARX and ARMAX model identifications were also carried out by using a standard prediction-error method [12. The ARX model has general structure of the form: $y[k]=\sum_{i=1}^{n_{a}} a_{i} y[k-i]+\sum_{i=1}^{n_{b}} b_{i} u[k-i]+e[k]$, whereas the general structure of the ARMAX model is $y[k]=\sum_{i=1}^{n_{a}} a_{i} y[k-i]+\sum_{i=1}^{n_{b}} b_{i} u[k-i]+\sum_{i=0}^{n_{c}} c_{i} e[k-i]$, where $e$ is a white noise process and $a_{i}, b_{i}, c_{i} \in \mathbb{R}$.

Parameters $n_{a}$ and $n_{b}$ were made equal to the true parameters of the system $\left(n_{a}=n_{b}=4\right)$. In this manner, the comparison with the wavelet-packet algorithm is carried out under favourable conditions for the ARX and ARMAX techniques. The sensor measurements were degraded with zero-mean white gaussian noise filtered by a $10^{\text {th }}$-order Butterworth bandpass filter. The ARMAX parameter $n_{c}$ was set to 10 accordingly.

Three cases were considered, with measurement noise in the ranges (3-5) Hz, (5-8) Hz, and (2-8) Hz, respectively. The noise was normalized in order to have the same root-mean-square value in all three cases. Figs. $3 \mathrm{a}, 3 \mathrm{~b}, 3 \mathrm{c}$, present the Fast Fourier Transform of a noise realization in each case. As can be seen, there is a significant overlap of the noise spectrum with respect to the system output shown in Fig. 2 ,

Fig. 4 depicts the wavelet-packet decomposition trees obtained according to the GCV criterion in the three noise cases. As can be seen, the tree with fewer nodes (Fig 4k) corresponds to the situation in which the noise was spread over a wider frequency region $(5-8 \mathrm{~Hz})$. It can be argued that the GCV algorithm automatically joined the subbands with poor signal-to-noise ratio in order to increase the amount of data employed in the identification of each subband 

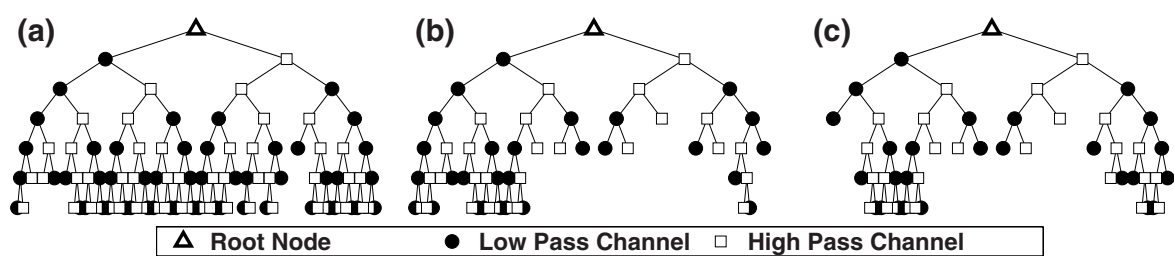

Fig. 4. Identification results: Wavelet-packet decomposition trees obtained for the three coloured-noise cases under study: (a) (3-5) Hz, (b) (5-8) Hz, (c) (2-8) Hz

(a)
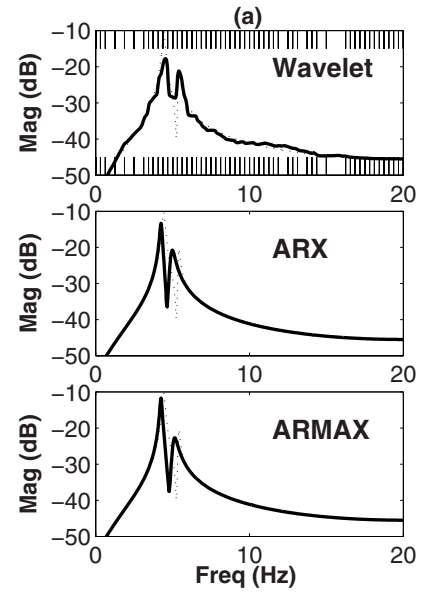

(b)
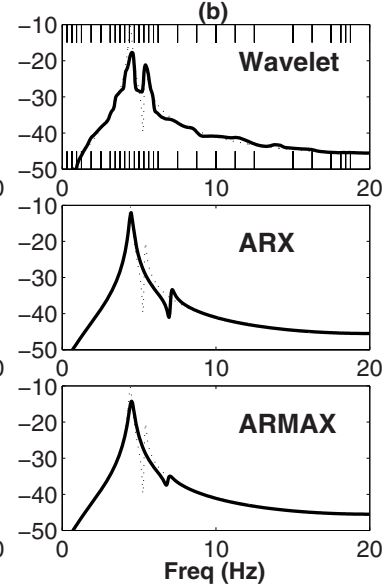

(c)
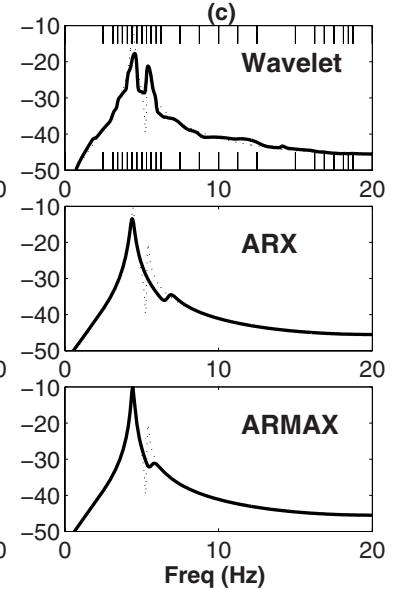

Fig. 5. Identification results: Wavelet-packet, ARX and ARMAX frequency responses obtained for the three coloured-noise cases under study: (a) (3-5) Hz, (b) (5-8) Hz, and (c) $(2-8) \mathrm{Hz}$. The dotted curves indicate the exact frequency response of the system. In the wavelet-packet models, the vertical lines indicate the frequency partitions.

model. In contrast, the tree with more nodes (Fig. 4 a) corresponds to the situation where the noise is concentrated on a frequency band $(3-5 \mathrm{~Hz})$ in which the plant output has the largest power, as shown in Fig. 2. In this case, joining subbands was not necessary because the signal-to-noise ratio was high throughout the entire spectrum.

Fig. 5 compares the frequency responses for the resulting wavelet-packet, ARX and ARMAX models. The graphs for the wavelet-packet models (top row) also indicate the subband partition of the frequency axis. As can be seen, wider frequency intervals are found in case (c), which is in agreement with the previous discussion concerning the decomposition trees. Fig. 5 also shows that the outcome of ARX identification is very sensitive to changes in the spectral features of the noise. Such a finding was expected because the ARX model structure assumes that the noise is white. The ARMAX results are slightly better, but the resulting model still fails to reproduce one of the resonance peaks in cases (b) 
and (c). In contrast, the wavelet-packet algorithm correctly identified the position of both resonance peaks in all cases. Moreover, the algorithm can be seen to be robust with respect to changes in the noise spectrum, as the identification results in (a), (b), and (c) are almost indistinguishable.

It is worth noting that the values of $n_{a}, n_{b}$ and $n_{c}$ for the ARX and ARMAX models usually need to be estimated from the identification data. Therefore, in a more realistic identification scenario, the advantages of the wavelet-packet technique could be even more evident.

\section{Conclusions}

This work reviewed a recently proposed technique for linear system identification in frequency subbands by using wavelet packets. In the proposed formulation, the wavelet-packet decomposition tree is used to establish frequency bands where subband models will be created. An optimization of the tree structure is performed by using a generalized cross-validation method in order to achieve a compromise between accuracy and parsimony of the overall model.

In an application example involving the identification of the longitudinal flexible model of a simulated aircraft in straight flight, the wavelet-packet technique was favourably compared with standard ARX and ARMAX identification techniques in terms of resonance peak identification and sensivity to coloured measurement noise. The present work shows that the wavelet-packet technique handles coloured noise in a natural manner by smoothing the identified frequency response in regions where the signal-to-noise ratio is poor.

Furthermore, it was observed that the wavelet-packet technique performs an adaptive partitioning of the frequency axis, which is an advantage of wavelet packets over the standard wavelet transform. It was shown that the proposed wavelet-packet technique leads to narrower frequency bands in the region of the frequency axis where the signal-to-noise ratio is larger.

Future works could adopt other basic structures for each subband model $\mathrm{M}_{i, j}$. For instance, more filter taps could be used in the FIR term. Furthermore, the use of autoregressive terms could also be investigated. Finally, it would be worth studying the possibility of extending the technique to the identification of a broader class of systems, such as piecewise-affine plants.

\section{Acknowledgements}

The authors acknowledge the support of Embraer, CNPq (research fellowship and postdoctoral grant 200721/2006-2) and FAPESP (grant 2006/58850-6). The valuable suggestions of Prof. Takashi Yoneyama (Instituto Tecnológico de Aeronautica) and Prof. Francisco Assis de Oliveira Nascimento (UnB - Universidade de Brasília) are also gratefully acknowledged. 


\section{References}

1. Abdelghani, M., Verhaegen, M., Van Overschee, P., de Moor, B.: Comparison study of subspace identification methods applied to flexible structures. Mechanical Systems and Signal Processing 12(5), 679-692 (1998)

2. Burcharles, A., Vacher, P.: Flexible aircraft model identification for control law design. Aerospace Science and Technology 6, 591-598 (2002)

3. Chen, H.X., Chua Patrick, S.K., Lim, G.H.: Adaptive wavelet transform for vibration signal modelling and application in fault diagnosis of water hydraulic motor. Mechanical Systems and Signal Processing 20(8), 2022-2045 (2006)

4. Coifman, R.R., Wickerhauser, M.V.: Entropy-based algorithms for best basis selection. IEEE Transactions on Information Theory 32, 712-718 (1992)

5. Cooper, J.E., Desforges, M.J., Wright, J.R.: Modal parameter identification using an unknown coloured random input. Mechanical Systems and Signal Processing 9(6), 685-695 (1995)

6. Dorfan, Y., Feuer, A., Porat, B.: Modeling and identification of LPTV systems by wavelets. Signal Processing 84, 1285-1297 (2004)

7. Erlicher, S., Argoul, P.: Modal identification of linear non-proportionally damped systems by wavelet transform. Mechanical Systems and Signal Processing 21(3), 1386-1421 (2007)

8. Hasan, M.K., Chowdhury, A.K.M.Z.R., Khan, M.R.: Identification of Autoregressive Signals in Colored Noise Using Damped Sinusoidal Model. IEEE Transactions on Circuits and Systems-I: Fundamental Theory and Applications 50(7), 966-969 (2003)

9. Ho, K.C., Blunt, S.D.: Adaptive sparse system identification using wavelets. IEEE Transactions on Circuits and Systems II - Analog and Digital Signal Processing 49(10), 656-667 (2002)

10. Huang, K., Aviyente, S.: Information-theoretic wavelet packet subband selection for texture classification. Signal Processing 86(7), 1410-1420 (2006)

11. Huang, C.S., Su, W.C.: Identification of modal parameters of a time invariant linear system by continuous wavelet transformation. Mechanical Systems and Signal Processing 21(4), 1642-1664 (2007)

12. Ljung, L.: System Identification: Theory for the User, 2nd edn. Prentice-Hall, Englewood Cliffs (1999)

13. Paiva, H.M., Galvao, R.K.H.: Wavelet-packet identification of dynamic systems in frequency subbands. Signal Processing 86(8), 2001-2008 (2006)

14. Vera-Candeas, P., Ruiz-Reyes, N., Rosa-Zurera, M., Cuevas-Martnez, J.C., LpezFerreras, F.: Sparse Approximations in Signal and Image Processing. Signal Processing 86(3), 432-443 (2006)

15. Vetterli, M., Kovacevic, J.: Wavelets and Subband Coding. Prentice-Hall, Upper Saddle River (1995)

16. Zheng, W.X.: Estimation of the Parameters of Autoregressive Signals From Colored Noise-Corrupted Measurements. IEEE Signal Processing Letters 7(7), 201-204 (2000)

17. Zheng, Y., Tay, D.B.H., Lin, Z.: Modeling general distributed nonstationary process and identifying time-varying autoregressive system by wavelets: theory and application. Signal Processing 81, 1823-1848 (2001)

18. Zheng, W.X.: Parametric Identification of Linear Systems Operating Under Feedback Control. IEEE Transactions on Circuits and Systems-I: Fundamental Theory and Applications 48(4), 451-458 (2001) 